\section{The Royal College of Ophthalmologists guidelines on serum eye drops for the treatment of severe ocular surface disease: executive summary}

\section{Introduction}

Serum eye drops (SED) are a useful adjunctive treatment for patients with severe ocular surface disease (OSD), especially those with a compromised tear film. Serum contains a large number of epitheliotrophic factors that are present in tears. These factors are likely to be responsible for the therapeutic benefits observed with SED therapy compared to conventional commercially available ocular lubricants. Prescribed and over-the-counter tear substitutes primarily alleviate symptoms through reduction of friction and shear-forces caused by blink-induced biomechanical trauma. This mechanism of action appears largely to be independent of structural chemistry and viscosity of the lubricant product. By contrast, SED provide a variety of nutritional molecules such as vitamins, glucose, growth factors, and immunoglobulins. These help to restore an environment that promotes reepithelialisation and supports ocular surface health.

SED are currently classified by the Medicines and Healthcare products Regulatory Agency (MHRA) as an unlicensed medicinal product (ie, hospital 'special'). The MHRA advises that anyone prescribing an unlicensed product must be satisfied that there is a special need for the unlicensed medicinal product, and that the unlicensed medicine should not be supplied where an equivalent licensed medicinal product can meet the special needs of the patient. This college guideline sets out recommendations and good practice points for the safe use of SED for the treatment of severe OSD. It aims to improve not only compliance with MHRA advice, but also standardise practice and improve patient morbidity. The following areas have been addressed:

- Patient groups that may benefit from the use of SED

- Clinical situations for the use of autologous SED (Auto-SED) and allogeneic SED (Allo-SED)

- SED formulation, frequency of therapy, and withdrawal

- Monitoring of treatment efficacy

The full guidance can be found online at: https://www. rcophth.ac.uk/standards-publications-research/clinicalguidelines / and https://www.nature.com/articles/ eye2017209 and a Quick Reference Guide for clinicians is provided (Figure 1). The criteria used for the grades of recommendations are found in Table 1 and a summary of recommendations are presented in Table 1a-f.

\section{Lay summary}

Ocular Surface Disease (OSD) is a global public-health problem. Severe dryness of the eye has significant impact on a person's physical, emotional, and social well-being.

The front of the eye is complex and has an outer surface known as tear film. A range of components contribute to how tears are made, what they contain and how they are distributed to keep the surface of the cornea smooth to enable sight and comfort. Failure of one or more of these complex components due to disease or injury results in dryness of the eye. In its severest form, OSD may lead to blinding complications.

Current practice for patients with ocular surface disease A patient with dry eye disease is treated in a stepped approach. When commercially available artificial tears do not provide relief and the patient does not respond to conventional treatments, the ophthalmologist might suggest that a patient with severe OSD might benefit from SED which are made from blood. Artificial tears made from blood have been shown to be effective because they contain many of the substances found in normal tears. They have been found to be superior to conventional treatment for improving ocular surface health and providing comfort.

Autologous SED (Auto-SED) are made from blood donated by the patient. Patients who are not suitable to provide an autologous donation can receive allogeneic serum drops (Allo-SED) which are made from blood donated by a male volunteer donor. SED are currently reserved for people who have severe disease who have not responded to standard intervention. They are also used for those who require supportive therapy for specialist ocular surgery or for management of ocular surface injury.

\section{The production of serum eye drops}

The NHS Blood and Transplant (NHSBT) has been providing SED since 2003. It is the only accredited production facility in the UK. NHSBT prepares SED from the patient's own blood (Auto-SED) and from individual (not pooled) male-volunteer blood donors (Allo-SED). To make the drops, the donated blood is processed to separate out the serum. Although there are variations in practice in other countries, in the UK, the serum is diluted with $50 \%$ saline and is transferred into sterile dropper bottles ready to be frozen. SED have a shelf life in the freezer of 12 months from the date of donation.

\section{The current situation and need for guidance}

Currently, SED is a highly specialised and high cost intervention for patients with OSD. The MHRA, the government body that regulates medicines and medical devices, classifies SED treatment as an unlicensed medicine. This means all licensed medical options should be considered by the doctor responsible for the patient before they are able to prescribe SED. There is geographical inequity in access to treatment that is currently being considered for exclusion from the National Tariff as a High Cost Drug. This Guidance aims to set out defined criteria for the use of SED, the monitoring of clinical and patient -reported outcomes and therefore improving patient care and safety while on treatment.

\section{Good practice points and recommendations relevant to patients}

Using the Royal College of Ophthalmologists' Guidelines Development Manual, a systematic review of literature has been carried out in order to focus on the best evidence available so that key questions may be addressed. 


\section{Quick Guideline Reference for the Use of Serum Eye Drops in Severe Ocular Surface Disease}
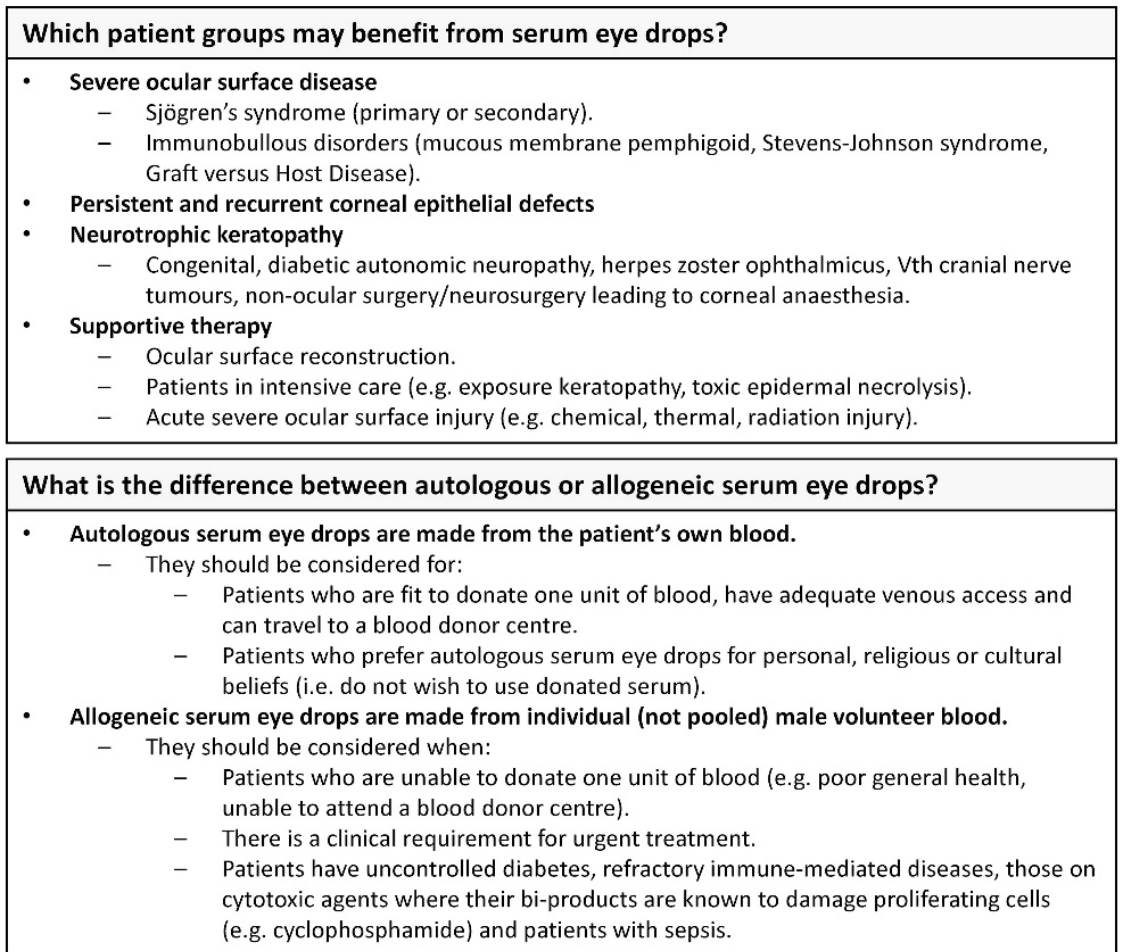

How should serum eye drops be prescribed?

- Serum Eye Drops are an MHRA unlicensed medicine ('special'), and should only be prescribed after licensed treatments have been considered or have been unsuccessful.

- Patient's disease severity should be defined with subjective and objective parameters, and be monitored throughout treatment to determine treatment response. It is recommended that monitoring occurs both locally and in a centralised registry.

- Autologous and Allogeneic Serum Eye Drops as a $50 \%$ dilution in $\mathbf{0 . 9 \%}$ Sodium chloride are recommended (as provided by the NHSBT, the only accredited production facility in the UK).

- Frequency and duration of treatment depends upon individual circumstances.

- Withdrawal and stopping strategies should be considered in all patients commenced on serum eye drops treatment before committing patients to indefinite treatment. For example:

- In ocular surface disease: Withdrawal of treatment after one year of therapy to define induction of remission before reinstating indefinite treatment if symptoms relapse.

- In persistent corneal epithelial defects: Withdrawal of treatment after surface of the eye has healed and restoring treatment if the surface shows signs of breakdown.

Figure 1 Quick reference guide for the use of autologous and allogeneic serum eye drops in severe ocular surface disease.

Recommendations affecting patients as key stakeholders may be summarised as follows.

- SED will benefit patients who have not responded or only partially responded to licensed interventions.

- When comparing the cost and clinical effectiveness of Auto-SED vs Allo-SED in the treatment of people with OSD, it is recommended that if a patient is unable to donate one unit of blood or a patient requires urgent treatment, Allo-SED are recommended.
- Published studies focus on concentrations of 20, 50, and $100 \% .50 \%$ is considered by NHSBT to be the best concentration for general use, although there are no internationally agreed standard procedures for the manufacture.

- There is no clear evidence regarding the duration of treatment or the effect of treatment with SED. It is recommended that treatment should either be for a defined period or there should be an appropriate point 
Table 1 Summary of recommendation grade

\begin{tabular}{|c|c|}
\hline Grade & Explanation \\
\hline A & $\begin{array}{l}\text { At least one meta-analysis, systematic review, or RCT rated as } 1++ \text {, and directly applicable to the target population; or } \\
\text { A body of evidence consisting principally of studies rated as } 1+\text {, directly applicable to the target population, and } \\
\text { demonstrating overall consistency of results } \\
\text { A body of evidence including studies rated as } 2++ \text {, directly applicable to the target population, and demonstrating overall } \\
\text { consistency of results; or Extrapolated evidence from studies rated as } 1++ \text { or } 1+\end{array}$ \\
\hline B & $\begin{array}{l}\text { A body of evidence including studies rated as } 2+\text {, directly applicable to the target population and demonstrating overall } \\
\text { consistency of results; or Extrapolated evidence from studies rated as } 2++\end{array}$ \\
\hline $\mathrm{C}$ & Evidence level 3 or 4 ; or Extrapolated evidence from studies rated as $2+$ \\
\hline GPP & Good practice points based upon consensual expert opinion where the evidence base does not support A-C grading \\
\hline MHRA & Medicines and Healthcare products Regulatory Agency Guidance Note $14^{\mathrm{a}}$ \\
\hline $\mathrm{R}$ & Further research is required in this area \\
\hline
\end{tabular}

${ }^{a}$ MHRA, 2014. The supply of unlicensed medicinal products ('specials') MHRA Guidance Note 14.

Table 1a MHRA Guidance Note 14 (2014), supply of unlicensed medicinal products ('specials'):

Recommendation Grade*

- Serum eye drops are an unlicensed medicine. The MHRA guidance note on the supply of unlicensed medicinal products MHRA ('specials') applies to delivery of this service.

- Note 2.2: Anyone supplying an unlicensed medicinal product, where an equivalent licensed medicinal product is available must be satisfied as to the existence of a special need for the unlicensed medicinal product. MHRA expects that documentary evidence of this special need should be obtained by manufacturers, importers or distributors and that this evidence should be made available on request of the Licensing Authority.

- Note 2.3: An unlicensed medicine should not be supplied where an equivalent licensed medicinal product can meet the special needs of the patient. Responsibility for deciding whether an individual patient has 'special needs' which a licensed product cannot meet should be a matter for the doctor responsible for the patient's care. Examples of 'special needs' include an intolerance or allergy to a particular ingredient.

${ }^{*}$ Refer to Table 1: Summary of recommendation grade.

Table $\mathbf{1 b}$ Serum eye drops should be considered in the following groups of patients:

- Patients who have refractory or partially responsive acute or chronic severe ocular surface disease where licensed interventions have been considered.

- Patients with other ocular surface conditions such as recurrent corneal erosions, persistent epithelial defects and limbal epithelial stem cell failure may benefit if licensed interventions have been unsuccessful.

- Supportive therapy such as for patients undergoing ocular surface reconstruction.

* Refer to Table 1: Summary of recommendation grade.

Table 1c Clinical situations where Autologous versus Allogeneic Serum Eye Drops should be considered:

Recommendation Grade*

- Autologous Serum Eye Drops (Auto-SED) should be considered for patients who are fit to donate one unit of blood, are GPP able to travel to a blood donor centre, or the patient prefers serum eye drops to be made from their own blood.

- Allogeneic serum eye drops (Allo-SED) should be considered in patients who are unable to donate one unit of blood such as those who are in poor general health, unable to attend a blood donor centre, less than age 16 years, or there is a clinical requirement for urgent treatment.

- Clinical trials comparing the clinical efficacy and cost effectiveness of Auto-SED vs Allo-SED are required.

*Refer to Table 1: Summary of recommendation grade. 
Table 1d Impact of the variability of individual nutritional constituents within supplied serum eye drops batches on clinical and patient outcomes

- Allo-SED should be considered as an option in patients with uncontrolled diabetes, refractory immune-mediated diseases, those on cytotoxic agents or where their bi-products are known to damage proliferating cells (eg cyclophosphamide) and patients with sepsis.

- Detailed serum constituent analyses of sequential donations from patient and healthy donors is required to interrogate biovariability of each donation and the impact this could have on ocular surface health.

- Further work on the development of protocols that reduce variability of biological constituents is required, for example, pooling of serum samples from multiple donors with measured ranges of main constituents.

${ }^{*}$ Refer to Table 1: Summary of recommendation grade.

Table 1e Concentration of formulation, duration and frequency of SED treatment for patients with ocular surface disease

- Auto-SED and Allo-SED as a $50 \%$ dilution in $0.9 \%$ sodium chloride is recommended (as provided by NHSBT, the only accredited SED production facility in the UK).

- Frequency and duration of treatment depends upon individual circumstances. The doctor responsible for patient care should consider withdrawal and stopping strategies in all patients commenced on SED treatment before committing patients to indefinite treatment. Such strategies may include (i) withdrawal of treatment after 1 year of therapy in patients with ocular surface disease, to define induction of remission before reinstating indefinite treatment if symptoms relapse, or (ii) in patients with persistent corneal epithelial defects, withdrawal of treatment after surface of the eye has healed and restoring treatment if the surface shows signs of breakdown.

- Further research is required on the optimal formulation and diluent. This includes considering whether a $100 \%$ formulation is as effective as one that is diluted. A search for vehicles or carriers that improve the retention time and patient satisfaction is recommended.

- Further work is required on the frequency and duration of serum eye drops treatment used for each clinical indication. Clinical trials should specifically consider when it might be safe to implement treatment withdrawal in patients who have achieved measured success or remission according to pre-set defined criteria.

*Refer to Table 1: Summary of recommendation grade.

Table 1f Monitoring of treatment response and progression of disease

- Instruments for assessment of the impact of treatment on health-related quality of life and objective grading of patient perceptions of disease using utility instruments specific for ocular surface disease, should be considered for use regularly in the clinical setting. These include the Ocular Surface Disease Index (OSDI) or the shorter Dry Eye Questionnaire - 5 (DEQ5).

- Consistent recording of clinical outcome measures and scoring of disease should be considered. This includes visual acuity, meniscus height, presence of filaments, tear film break-up time, ocular surface staining score, for example, Ocular Staining Score, epithelial defect measurements (if present) and Schirmer's test without anaesthetic.

- It is advised that patients treated with Auto-SED and Allo-SED should be enrolled into a national programme. Frequency and duration of treatment together with serious adverse events should be recorded using a standard reporting procedure. A minimum follow-up of 6 months and then annually should be considered.

- Development and validation of SED-specific patient reported outcome tools and minimal clinical data sets for efficient

(1)
outcome reporting is required.

${ }^{*}$ Refer to Table 1: Summary of recommendation grade.

when it is stopped in order to assess the outcome. Patient- reported outcomes are an essential tool.

\section{Monitoring}

It is recommended that patients treated with Auto-SED and Allo-SED should be enrolled on a national programme of outcome reporting that include patient reported outcomes. Reports should include: frequency and duration of treatment and serious adverse events and reactions. Attempts to withdraw treatment and duration of remission should be recorded.

\section{Conflict of interest}

Dr A Chandrasekar and Dr R Lomas represented the NHS Blood and Transplant Tissue Services (accredited 
suppliers of the serum eye drops service in the UK) in the Guidelines Development Group. The remaining authors declare no conflicts of interest.

\section{Author contributions}

The multidisciplinary team involved in producing these guidelines was chaired by Miss Saaeha Rauz (Consultant Ophthalmologist specialising in ocular surface disease) who proposed and led the development of the guidelines. Miss Su-Yin Koay was involved in the grading of the evidence and providing summary tables. Mr Barny Foot represented the Royal College of Ophthalmologists Quality Team that supported the guidance development. Professor Stephen Kaye and Professor Francisco Figueiredo provided Ocular Surface Specialist clinical input and Mr Michael Burdon provided general ophthalmology input and assured the guidance was produced in accordance to the Royal College of Ophthalmologists Guidelines Development Manual. Dr Akila Chandrasekar and Dr Richard Lomas represented NHS Blood and Transplant Tissue Services and provided source data from the SED service. Mrs Elizabeth Dancey contributed as a Patient and Carer representative and heads the Serum Eye Drops Patient Focus and Support Group.

\section{Acknowledgements}

The Royal College of Ophthalmologists would like to thank the Cochrane Eyes and Vision Group and in particular Iris Gordon for her assistance in searching the evidence base. The development of this guideline was funded by The Royal College of Ophthalmologists. This guidance was peer-reviewed through a formal consultation process where members of the Royal College of Ophthalmologists, Bowman Club, Ocular Tissue Advisory Group and British Society of Blood and Marrow Transplant were invited to provide comments. The draft guidance was available for wider consultation through publication on the Royal College of Ophthalmologists website, and final version was ratified through the Scientific Committee at the Royal College of Ophthalmologists.

S Rauz ${ }^{1}$, S-Y Koay ${ }^{2,3}$, B Foot ${ }^{2}$, SB Kaye ${ }^{4}$, F Figueiredo ${ }^{5}$, MA Burdon ${ }^{2,6}$, E Dancey ${ }^{7}$, A Chandrasekar ${ }^{8}$ and R Lomas ${ }^{8}$

${ }^{1}$ Birmingham and Midland Eye Centre and University of Birmingham, Birmingham, UK

${ }^{2}$ Royal College of Ophthalmologists, London, UK

${ }^{3}$ Moorfields Eye Hospital, London, UK

${ }^{4}$ Royal Liverpool University Hospital, Liverpool, UK

${ }^{5}$ Newcastle Royal Victoria Infirmary, Newcastle, UK

${ }^{6}$ Queen Elizabeth Hospital Birmingham, Birmingham, UK

${ }^{7}$ Serum Eye Drops Patient Focus and Support Group, Birmingham, UK

${ }^{8} \mathrm{NHS}$ Blood and Transplant Tissue Services, Liverpool, UK E-mail: s.rauz@bham.ac.uk

Eye (2018) 32, 44-48; doi:10.1038/eye.2017.208;

published online 17 November 2017 